\title{
CONTRIBUTIONS TO THE FUNCTIONAL NEUROANATOMY \\ OF MORPHOSYNTACTIC PROCESSING IN L2
}

\author{
Ruth De Diego-Balaguer ${ }^{1,2}$ \\ Antoni Rodriguez-Fornells ${ }^{1}$
}

${ }^{1}$ ICREA, University of Barcelona, Dep. of Physiological Sciences II and IDIBELL, Faculty of Medicine, L’Hospitalet de Llobregat, Spain

${ }^{2}$ INSERM U955, Interventional Neuropsychology, IM3-Paris 12, Créteil and Paris, France, and Département d'Etudes Cognitives, Ecole Normale Supérieure, Paris, France

Corresponding author:

Ruth de Diego Balaguer, PhD

Universitat de Barcelona

Facultat de Medicina

Departament de Ciències Fisiologiques II

Feixa Llarga, s/n

08907 L'Hospitalet de Llobregat (Spain)

Email: ruth.de.diego@gmail.com 
Studies about bilingualism and second language acquisition have a long tradition within linguistic and psycholinguistic research. With the global population becoming more and more multilingual and the recent proliferation of research in cognitive neuroscience, an increasing number of studies examining the way our brain is able to learn, represent, and handle more than one language at the same time are currently available. But few attempts have been made to transpose psycholinguistic models of second language acquisition (SLA) into functional neuroanatomic models. An important problem that arises when pursuing this goal is partially due to the delay in the development of cognitive neuroscience of language compared to psycholinguistics. In general, neurolinguistic models focus on very broad and general questions about bilingualism, while psycholinguistic research is already at the stage of addressing more specific and fine-tuned questions. This Granularity Mismatch Problem (Poeppel \& Embick, 2005) in the degree of zooming into this research topic is not exclusive of L2 research, but it is present in language research in general (Hauser \& Bever, 2008). In either case, it often becomes difficult to put together the results from these different perspectives into one integrated model.

This special issue of the journal is concerned with the representation of morphologically complex words in L2. This aspect is particularly interesting because, although it taps into the lexicon, it can provide information regarding the types of computations that L2 speakers perform to comprehend and produce words involving grammatical information as well. There is a common agreement in the field that, in general, L2 learners find grammatical aspects particularly difficult compared to lexical-semantic information in the second language, especially when exposure to this new language occurred after puberty (Birdsong \& Molis, 2001; Johnson \& Newport, 1991; Weber-Fox \& Neville, 1996). Therefore, it is likely that in the case of morphologically complex words, L2 speakers would tend to remember the full forms of words instead of applying grammatical 
computations. This functional distinction between lexical and grammatical knowledge also appears in L1. In learning a first language, words of the lexicon are first produced as invariable chunks, and productive rules are applied to them later during development (Clark, 1998; Tomasello \& Brooks, 1999). Through the study of brain-damaged patients, selective impairments in acquisition and retrieval of lexical/semantic information or grammar have been identified and described (Miozzo, 2003). In the same way, several neuroimaging studies have shown the recruitment of different brain areas for regular and irregular inflected forms (Beretta et al., 2003; De Diego-Balaguer et al., 2006).

Different theories offer explanations for these apparent dichotomies in the morphosyntactic domain. Although most models were initially developed to describe native language acquisition and processing, they may also offer explanations for the pattern of development observed in SLA (Rodriguez-Fornells, Cunillera, Mestres-Misse, \& De DiegoBalaguer, 2009; De Diego-Balaguer \& Lopez-Barroso, 2009). Despite the fact that these models come from different theoretical perspectives, they propose very similar brain structures as responsible for morphosyntactic processing, encompassing the prefrontal cortex (PFC, including the inferior frontal gyrus, IFG) and temporal lobe regions (including, middle and superior temporal gyrus; MTG and STG, respectively). These brain regions described overlap across the different models and can be further complemented by taking into account learning models from other non-linguistic domains. However, these proposals differ in the description of the dynamics and connectivity of these networks. Some of them dissociate the temporal and frontal networks, while others include subcortical structures that have different roles in morphosyntactic compositionality. Finally, other theoretical positions link frontal and temporal areas within the same connected network. In addition, these models also differ with respect to the nature of the computations that are carried out within these networks. This functional information would also help to explain the dynamical relations among the different 
brain areas and the possibility that cognitive functions other than language, such as attention, working memory, and executive functions, could affect various aspects of SLA in the course of learning.

The contributions from psycholinguistic research are crucial to the improvement of neurolinguistic models. This importance stems from the fact that psycholinguistic research is posing more specific questions than those in many current cognitive neuroscience studies. For example, in the present issue we have several examples of the type of questions that psycholinguistic research can raise. In general, most of the work on morphosyntactic research from neuroscience and psycholinguistics has come from studying English, a language that has a relatively simple morphological system. However, the picture becomes more intricate when these models are extended to more complex morphological systems. The contributions in this journal issue embrace the complexity of different languages both in the role of the L1 background and from L2 processing perspective. They describe results in perception and production, and they study different aspects of morphology with unique types of instruction (Table 1), giving a complete and enriched overview of the field. In the following commentary, we will briefly introduce the most important neurofunctional proposals relevant to L2 morphosyntactic acquisition and processing, and we will discuss the implications of the results provided in this special issue for these models.

\section{Models dissociating frontal and temporal networks}

Based mostly on studies comparing regular and irregular verbs in different languages, dual-system accounts (Clahsen, 1999; Pinker, 1999; Ullman, 2001b) propose that words and rules of language are clearly different entities requiring specific mechanisms of their own to be acquired and processed. The interest in studying the contrast between regular and irregular forms in order to tackle the grammar-lexicon distinction resides in the possibility of matching 
these two aspects, which would otherwise require the comparison of very different materials (sentences vs. single words). Regular forms are single words, but they share the same stem and apply different affixes according to the context in which the word appears, thus theoretically requiring the application of grammatical rules. Irregular forms, on the contrary, display idiosyncratic phonological variations that depend on the specific item from which the form is derived. Therefore, their forms would need to be lexicalized.

Within this framework, one clear instance of the anatomo-functional transposition of a psycholinguistic model comes from the Declarative/Procedural Model (Ullman, 2001b; Ullman, 2001a), which had a great impact in the field of L1 and L2 morphological processing. Its starting point is the psycholinguistic proposal that regular forms are indeed acquired by the extraction of a rule that is later applied to all verbs by default. This is the case in languages like English in which only one regular pattern exists. This proposal seems more difficult to apply to languages with richer morphological systems that include different suffixes for each conjugation class and that therefore appear to contain more than one regular pattern. Clahsen and collaborators (Sonnenstuhl et al., 1999) have proposed that, in those languages, such as German, only one rule (one conjugation class) is a default rule that is processed compositionally, while the others are accessed as whole-forms. Within this framework, the results of Bowden et al.'s (this issue) study on Spanish, a morphologically rich language with three conjugation classes, support this possibility. In contrast, irregular verbs are acquired and produced using an associative network that picks similarities between the different related forms (Pinker, 1999). Retrieving these forms blocks the application of the default rule that would otherwise be applied. The anatomo-functional counterpart of this model takes a more domain-general view (Ullman, 2001b), stating that regular verbs require the acquisition and application of routines and thus engage procedural memory handled by a fronto-striatal circuit (Figure 1A). This circuit is usually involved in the acquisition of new skills and their 
execution from other domains, such as motor and cognitive sequences (Koechlin, Danek, Burnod, \& Grafman, 2002). The basal ganglia refer to a set of grey matter subcortical structures localized deep in the brain that are connected to several areas throughout the brain in parallel loops holding different motor and cognitive functions (Alexander et al., 1986; Middleton \& Strick, 2000). The striatum is one of the primary components of the basal ganglia receiving inputs from different regions of the cortex. The projections from the striatum reach the cortex through the thalamus.

On the other hand, because irregular verbs have idiosyncratic forms, they have to be stored in their full form by declarative memory during acquisition. They are then retrieved from long-term memory when they have to be produced. Declarative memory is responsible for the explicit acquisition of new information and involves a temporo-parietal network that is dissociable from the one subserving procedural memory (Milner, Squire, \& Kandel, 1998; Stern et al., 1996), which is usually associated with implicit processing (Figure 1A).

\section{Declarative-procedural memory and its relation with SLA}

According to this model, SLA differs from learning a native language because explicit instruction and intentional strategies engage declarative memory for both lexical and grammatical acquisition (Ullman, 2001a). Through intense practice, declarative acquisition of grammatical information may become procedural in the long run. As is the case for other cognitive skills (Anderson, 1987), this automatization of explicitly learned morphosyntactic rules seems to gradually progress rather than suddenly shift from controlled to automatic processing, at least in artificial language learning (DeKeyser, 1997). Although some authors have shown that procedural learning is skill-specific, and that transfer is limited between perception and production, others have shown that training in one modality can clearly transfer across domains (Hoen et al., 2003; Marcus, Fernandes, \& Johnson, 2007). Transfer is 
an intrinsic characteristic of procedural knowledge, and data gathered outside the language domain have shown that the striatum seems to be a key structure for the process of transfer abilities (Dahlin, Neely, Larsson, Backman, \& Nyberg, 2008).

According to Ullman (2001a), another reason for the shift from procedural to declarative memory in L2 learning, compared to L1 learning, comes from the maturational constraints of the procedural network in the course of development. While a fronto-striatal pathway was initially related only to procedural memory and grammatical acquisition, more recent versions of the model (Ullman, 2006) introduce another fronto-striatal loop associated with declarative memory, adding a functional dissociation between the anterior and posterior subregions of Broca's area and the corresponding subcortical structures involved in declarative and procedural memory processing. The hypothesis for this functional dissociation is rooted in the cytoarchitectonic and connectivity differences between these areas (Amunts et al., 1999). It is also based on the integration of evidence from language and other cognitive tasks indicating that the anterior circuit, including the anterior portion of the inferior frontal gyrus (pars triangularis, BA 45), is implicated in the retrieval of lexical/semantic processing sustained by declarative memory. In contrast, the more posterior region and its connections (pars opercularis, BA 44) are hypothesized to be involved in procedural learning. According to Ullman, procedural learning is the common denominator of the functions reported to require this area. Examples of such functions are syntax and phonology, sequencing, working memory, and temporal processing. This more recent specification resembles other perspectives presented in the next section that propose a common processing route for language rule extraction and sequence learning in other domains, which might also require similar timing and working memory demands.

Aside from the underlying memory systems described in this model, this latter functional dissociation between lexical retrieval and suffixation (rule application) within the 
PFC complements the fronto-temporal dissociation initially proposed by Ullman et al. (2001a). Furthermore, it is also in line with the proposal derived from neuroimaging results from a study on Spanish, a language that is morphologically richer than English (De DiegoBalaguer et al., 2006). In Spanish, both regular and irregular verbs contain shared suffixes; however, irregular forms have different stems depending on the tense and person to be produced. In this language, a clear dissociation was observed within the PFC for the lexical retrieval of the specific stem needed for irregular verbs (Figure 1C) compared to the retrieval of the grammatical features needed for the inflection of both regular and irregular verbs. These regions of the PFC were also different from those used in the maintenance of the stem used for several forms of regular verbs.

Subcortical involvement and the role of executive functions in language learning

This recent refinement in the neuro-functional specification of the declarative/procedural model arises from the interest in differentiating between the processes and networks related to the application of consolidated knowledge and to its acquisition. Other proposals that are more interested in the learning process try to combine psycholinguistic evidence in speech perception with our knowledge from sequence learning in different domains, highlighting the important role of the fronto-striatal circuit in this type of learning (Dominey, Hoen, Blanc, \& Lelekov-Boissard, 2003; Lieberman, 2000). Because language learning requires the detection of sequential relations at the level of phonemes (phonotactics), syllables (word segmentation), and grammatical categories (syntax), it has been proposed that neural circuits related to sequential learning should have a central role in the extraction of dependencies from speech, including morphosyntactic information (Dominey et al., 2003; Lieberman, 2000; Ullman, 2006). Indeed, the striatum may have a greater role during acquisition of rules than during their later application once consolidated 
(de Diego-Balaguer \& Lopez-Barroso, 2009). During the acquisition of simple rules in monkeys, simultaneous intracellular recordings in the striatum and in the PFC show an initial burst of activation in striatal cells followed by a progressive response in the PFC, which is correlated with performance improvement (Pasupathy \& Miller, 2005). In humans, basal ganglia abnormalities are described in children with difficulties in language development (Liegeois et al., 2003; Teicher et al., 2000), whereas subcortical lesions in adults lead to better prognosis and faster language recovery than cortical lesions (Hillis et al., 2004).

The reasons for this functional differentiation, with a greater involvement of subcortical structures in the earlier stages of acquisition, remain unknown. However, this differentiation is supported by the fact that subcortical structures are also crucial in consolidated language in order to deal with aspects demanding cognitive control, such as ambiguous sentences, syntactic violations, or the application of non-default rules (Kotz et al., 2002; Teichmann et al., 2005; Wahl et al., 2008; Munte \& Kutas, 2008). Thus, in these cases where automatic processing is blocked and cognitive control is required, the striatum has a prominent role. This is consistent with the greater role of this structure in the course of acquisition because rules are not yet automatized at that stage (De Diego-Balaguer et al., 2008). The weight of executive control in the learning process is an important point that may partly explain the individual differences observed in SLA. Dominey et al. (Dominey, Inui, \& Hoen, 2008; Dominey et al., 2003) have presented a more formalized model with a connectionist proposal including the anatomical counterpart for each proposed module. This model was not developed to understand SLA. Therefore, it does not include important aspects necessary to explain this complex learning problem. For example, modulatory influences, such as explicit and implicit instruction, are absent as variables that may affect the dynamics of the model. However, the model incorporates the influences of other cognitive functions, namely, the importance of working memory for the acquisition of this type of information. 
Along these lines, in a recent study, patients with striatal degeneration (Huntington's disease patients) (De Diego-Balaguer et al., 2008) were presented with an artificial language containing words with rules resembling morphosyntactic dependencies (e.g., bagoli, baseli as in is playing, is making). Their rule generalization capacity was specifically correlated with working memory, while vocabulary learning was correlated with episodic memory scores.

Similarly, in this current issue, Kempe and colleagues study the acquisition of Russian gender categories in a sample of native English speakers (Tables 1 and 2). After four training sessions spanning ten days and including the assessment of production and perception measures of gender marking, they assessed the influence of other cognitive functions on L2 outcome. In this paper, two measures of working memory were used in addition to measures of non-verbal intelligence. The Reading Span test (Daneman \& Carpenter, 1980) demonstrated a predictive value for incidental learning of L2 vocabulary, whereas the Nonword Span (Baddeley, Gathercole, \& Papagno, 1998) showed no influence when executive functions and general intellectual abilities were partialled out in the regression analyses. Interestingly, this inconsistency led the authors to propose that the susceptibility to interference that remains in the Reading Span measure when working memory and executive functions are controlled might facilitate learning associations between the new words and their meanings. This speculative interpretation would suggest not only that bilingualism in the long run would enhance resistance to interference (Costa, Hernandez, \& Sebastian-Galles, 2008), but also that subjects with lower susceptibility to interference would be more gifted for SLA a priori. Working memory and non-verbal abilities also influenced the individual differences for SLA. Nevertheless, this effect was not specific to the acquisition of gender categories. It influenced the overall performance in gender categorization as well as vocabulary acquisition. 
Current researchers interested in learning outside the language domain have done extensive work in investigating explicit and implicit learning in the domain of motor sequencing and categorization. This type of research has identified the brain networks involved in implicit and explicit learning, and indeed, there is great overlap between the networks involved in these two types of learning (Figures 1A and B). However, interesting differences have been identified. A greater weight has been put on subcortical structures in implicit learning, and there seems to be greater prefrontal involvement when explicit learning is achieved (Ashby \& O'Brien, 2005; Filoteo, Maddox, Salmon, \& Song, 2005; Fletcher et al., 2005). Although the declarative-procedural distinction is not equivalent to the explicitimplicit division, it is interesting for SLA that, in this context, there is evidence supporting the idea that these two neural systems (fronto-striatal and temporal) and learning procedures (declarative-procedural) are complementary and that they dynamically interact in a cooperative/competitive way during the course of learning. Note that, although both declarative memory and procedural memory are generally presented as dissociable networks (Figure 1A), these networks clearly overlap with those proposed for the explicit and implicit systems that are presented here as interactive and in a unified network (Figure 1B). The striatum is proposed as the key structure for these interactions because it is interconnected both to the medial temporal lobe and to the prefrontal cortex (Yin \& Knowlton, 2006). A competitive relationship has also been hypothesized because both systems work in parallel during learning. Initially, there is a greater reliance on the declarative/explicit systems, and, as a function of the input or the task, or when this type of learning fails, the procedural/implicit system is reinforced and further strengthened with practice (Ashby \& Valentin, 2005). This interaction leads to interesting possibilities, which fit well with the progression described in 
some studies from controlled memorization (declarative memory), which is generally explicit, to automatization (procedural memory), which is generally implicit (DeKeyser, 2000).

In fact, most situations involve both declarative and procedural learning. The amount of variability in the exemplars during the training phase, and the ease of memorizing associations might determine the weight of one type of learning over the other. This idea may also apply to language learning experiences. For example, the classical pattern of errors in L1 morphosyntactic development is described as following a U-shaped performance curve, with initial accurate production of regular and irregular forms followed by an over-regularization of all forms before the final correct performance (Plunkett \& Marchman, 1991). However, the results from Morgan-Short et al. (this issue) emphasize that, in L2 acquisition, this developmental progression might only appear in implicit learning. Two groups of subjects were implicitly or explicitly trained to acquire gender agreement in an artificial language (BROCANTO2). Behavioral measures and on-line event-related brain potentials (ERPs) were recorded at two different time-points in a longitudinal design including low and high proficiency stages. No difference between the implicit and explicit groups appeared during the judgment of sentences with agreement violations. However, differences were evident between the two ERP longitudinal evaluations. For noun-adjective and noun-article gender violations, the explicit group showed ERP modulations only at the high proficiency stage. In contrast, the implicit group showed a clear pattern of development across time, displaying an N400 modulation and a late negativity at the low proficiency stage, which evolved into a P600 response at the high proficiency stage. The N400 is a negative ERP component appearing at 300-400 ms, which is sensitive to various lexical and semantic/conceptual factors (semantic congruency, semantic priming, lexicality, word frequency, phonological priming and morphological properties of words) and in relation to word, sentential and discourse levels (Kutas and Federmeier, 2000). It shows a typical central-parietal distribution, with a right 
hemisphere preponderance, although in some experimental conditions, more frontal and anterior distributions have been observed. The P600 component is a positive component appearing at 500-600 ms, showing a posterior central-parietal distribution. It is normally elicited by syntactic violations and unexpected (complex) syntactic constructions such as sentences with noncanonical word order (Matzke, Mai, Nager, Rüsseler, \& Munte, 2002), and it has been associated with syntactic reanalysis (Kaan, Harris, Gibson, \& Holcomb, 2000). The Morgan-Short et al. study (this issue) highlights the importance of carrying out longitudinal studies in language learning in order to understand the dynamics of the learning process (Osterhout, McLaughlin, Pitkänen, Frenck-Mestres, \& Molinaro, 2006).

The overall pattern of results in the presented studies is in agreement with previous research indicating that the way a language is acquired may determine the way this language is represented and accessed. Nevertheless, it is also worth pointing out that all of the studies mentioned do not include near-native bilinguals (Birdsong, 2006; Hyltenstam \& Abrahamsson, 2000; Montrul \& Slabakova, 2003). It is also possible that, at this level of expertise, the implicit system could override explicit functioning by automatization and practice.

Finally, it is interesting to point out that, as proposed in the categorization domain (Ashby et al., 2005), the intrinsic characteristics of the morphological categories (gender, different plural suffixes, etc.) in the L2 may determine which type of learning (declarative/procedural) will be more suitable for successful acquisition. In that sense, in their comment in this issue, Clahsen et al. propose that some intrinsic factors make a grammatical category harder to learn than others, such as semantic complexity and transparency of the form-meaning relationship. These characteristics might determine the tendency of L2 learners to rely on declarative memory. From a review of the literature, Clahsen et al (this issue) state that domain-general factors such as poor decoding abilities, slower processing speed, 
computational resource limitations, and L1 background also play a role. However, these factors are insufficient to explain L1-L2 differences. They offer two possible explanations for the greater difficulty in learning morphosyntactic agreement, compared to case in SLA of English. Agreement is marked by bound affixes, while case is denoted by regular suppletive forms. Thus, one explanation resides in the tendency to store the word-forms in memory. The other explanation is also derived from the intrinsic characteristics of the two categories, with agreement spanning distant elements in the clause, which causes greater difficulty in L2 than in the object case, where dependencies are local in the verb phrase. Again, in the two explanations, the tendency to use declarative memory for agreement might be due to the inherent characteristics just described. For example, from Ellis's (2008) point of view, these characteristics might trigger attention in different ways, with suppletive forms/local dependencies more easily capturing attention and making agreement more prone to be stored in declarative memory as a whole form, compared to case.

\section{The dorsal-ventral route in language processing}

Along a related viewpoint emphasizing interactive dynamics between brain areas instead of dichotomic alternative networks, Marslen-Wilson and Tyler (2007) agree with the idea of decomposition of morphologically complex words, which implies that access to the lexicon is via the stem. However, their view does not assume a difference in the computations for simplex and complex words. Although acknowledging the implication of temporal and frontal networks in word processing, they adopt the distinction between the dorsal and ventral streams connecting these two areas, as documented in the monkey auditory system and later transposed to humans by Hickok and Poeppel (2007, 2004). Within this framework, the dorsal stream connecting the two areas via the arcuate fasciculus is responsible for morphological decomposition of all potentially decomposable words. At the same time, the ventral pathway 
is engaged in semantic interpretation of words. Thus, lexical processing is both "memorized" and "computed," and therefore, the dissociation in terms of declarative and procedural memory is orthogonal to their distinction.

Importantly, the decomposition mechanism that Marslen-Wilson and Tyler (2007) propose seems to apply in an automatic fashion during early processing. This means that, in SLA, if decomposition is blind and automatic when processing an L2 with the same morphological affixes, transfer should act automatically. Indeed, Scheutz and Eberhard (2004) found that native German speakers learning English activate the masculine gender when processing English words containing the agentive marker -er. However, this automatic decomposition might be modulated by executive control. Marslen-Wilson et al. (2007) suggested that frontal control processes should coordinate the use of the dorsal and ventral streams. This point is particularly relevant for SLA because it has been shown that executive control is necessary in bilinguals to manage the use of their two languages (Abutalebi, 2008; Bialystok \& Martin, 2004; Costa et al., 2008; Rodriguez-Fornells, Rotte, Heinze, Nosselt, \& Munte, 2002). Automaticity of decomposition is helpful when similarities between languages correspond to the same computations (i.e., the - s plural suffix might be used in different languages) and needs to be controlled when similarity is misleading and may cause errors [i.e., false friends between languages: red (the color in English)/red ("net" in Spanish)]. It remains to be studied whether we shape our executive control and the use of one stream or the other over learning as a function of what is shared and helpful in the processing between languages at the different levels of representation (phonological, morphological, semantics) (Rodriguez-Fornells, De Diego-Balaguer, \& Munte, 2006).

In this regard, Clahsen and collaborators have proposed that not all the conjugation classes are processed by decomposition, even in languages with rich morphological systems. Only one conjugation seems to behave as a default in German (Sonnenstuhl, Eisenbeiss, \& 
Clahsen, 1999). The default class is always characterized by full regularity in terms of the absence of phonological changes in the stem and the suffix applied, while non-default classes have changes in the stem of some tenses and persons, despite displaying a few regular forms. From the ventral-dorsal perspective (Marslen-Wilson et al., 2007), the exclusive decomposition of the default class in those studies might be derived from the fact that automatic parsing could not be performed, due to the absence of phonological transparency or productivity in some cases of the verb paradigm. In those cases, executive control might then send the information to the ventral stream. Bowden et al. (in this issue) have provided similar evidence for Spanish. In this study, participants performed a lexical decision task with verbs in different conjugation classes (default vs. non-default) presented in their regular form and in forms containing regular suffixes with irregular stem transformations. The results showed that both the conjugation class (default or non-default) and the irregularity of the stem influenced the compositionality of the forms as reflected in the response latency in a production task. All forms showed frequency effects indicating storage of the full-form, except for the default class in the forms without stem transformation. This default class was also fully stored for L2 learners, at least at their level of experience with the language ( $\sim 9$ months).

The work by Gor and Cook (this issue) is of specific relevance in this context because it related this aspect to that of implicit and explicit training of the L2. In their study, the authors compared L2 speakers with heritage speakers. Heritage speakers use their L1 at home, but this L1 is different from that of the community where they live. Thus, their proficiency is comparable to the L2 participants studied, although they are comparable to L1 speakers because they learned that language early through naturalistic and extensive language exposure. Gor and colleagues studied the influence of allomorphy, along with the effect of the frequency of the conjugation pattern, one aspect that was not considered in the Bowden et al. study (this issue). Both allomorphy and frequency of the conjugation pattern influenced the 
processing time in L1 and L2. However, a lack of decomposition was observed in the heritage group when compared to the L2 group. Thus, the different results seem to give a convergent picture with greater reliance in the ventral stream (semantic pathway) for heritage speakers lacking explicit instruction. While displaying the same proficiency as L2 speakers, they did not show the sensitivity to morphological complexity that the L2 group did. However, to what extent decomposition of the default class can be reached at high proficiency and with high levels of L2 experience remains unknown. In addition, the way this default class is created during the course of learning and in relation to the issues just mentioned is a question that remains to be answered in the future.

\section{Single-system accounts: Phonological and semantic overlap}

Other accounts propose that the same mechanism is applied to produce all types of words, and apparent morphological relations are an epiphenomenon of the phonological and semantic overlap that characterizes these related forms (Joanisse \& Seidenberg, 1999; McClelland \& Patterson, 2003). In particular, in Joanisse and Seidenberg's proposal (1999; Seidenberg \& Joanisse, 2003), forms are related according to their semantic and phonological overlap. Their model explains the dissociations reported by dual system accounts in brainlesioned patients (Ullman et al., 1997) using damage in the units coding for this information. Thus, their model has an indirect neuroanatomical basis. In their approach, regular and irregular forms are not qualitatively different; rather, they differ gradually, as noticed in some sub-regularities occurring in subgroups of irregular verbs (i.e., throw-threw, grow-grew, know-knew, etc.). Because regular verbs are systematically similar from a phonological point of view, lesions to phonological information in the model should give rise to problems with regular verbs. In addition, irregular verbs, which depend heavily on their semantic relations, should develop from lesions in the lexico-semantic module. From this perspective, lesions in 
the fronto-striatal circuit that induce problems in applying the correct morphological suffixes are due to a phonological impairment. In contrast, a lexico-semantic deficit should be correlated with temporal lesions in patients displaying difficulties with irregular verbs.

\section{Transfer as a function of L1-L2 similarity}

From this input-driven perspective, the learning process of L1 and L2 is achieved through the same neural system. Because the functioning of this system depends heavily on the input provided, the processing differences between L1 and L2 should greatly depend on the dissimilarities across the two languages. Therefore, these processing differences should arise from: i) disparities in the input characteristics as well as the amount of exposure, and ii) interference of L1 that has already shaped the dynamics of the network. Thus, difficulties in L2 are likely due to interference of L1 in terms of neural commitment (Bates, Wulfeck \& Mac Whinney, 1991; MacWhinney, 2002).

Concerning the first point, the data by Murphy and Hayes (this volume) illustrate how the absence of L1-like effects in L2 learners can be due to insufficient exposure to the characteristics of the language. Their starting point is the observation that native speakers of English tend to omit regular plural suffixes within noun-noun compounds with a headcomplement relationship (i.e. taxi - driver). In their study, the pattern of results in L1 speakers shows that this preference is influenced by the input inducing L1 speakers to obey the constraints that characterize the language. Namely, the language has the semantic constraint that items preceding a noun are not marked for plurality, along with the phonetic constraint that words ending in $-\mathrm{s}$ rarely precede nouns. By testing possessives that are semantically and morphologically singular but phonetically plural, the authors show that the distinction between regular and irregular plurals in compounds is learned from the general properties of the languages and not only from exposure to examples of compounds. The amount of 
exposure in L2 learners is not sufficient to extract this general pattern. It is worth mentioning here the results of Gor and Cook (this volume), who studied heritage speakers. Although this population was comparable to L1 speakers in their early exposure to the language, their impoverished exposure led to a lack of decomposition. These results highlight the importance of the amount of exposure in the representation of morphologically complex words, despite an early age of acquisition.

Concerning the second point, it has been proposed that, as a result of extensive exposure to L1, the system tunes attention to the relevant characteristics of this language (Ellis, 2008). The position of this attention filter with regard to the second language will determine the ability to transfer and learn this new language. Otherwise, the pre-wired system will overlook the relevant features when learning L2 morphology. According to this proposal, the combination of different factors, such as salience of the morpheme and degree of regularity, determine how easy this morphophonological rule is going to be learned. Subjective salience of one cue may not be the same in L1 and L2. In fact, as reported by Kempe et al. (in this issue), when the L1 background is a highly inflected language, L2 learners tend to focus more on word-form regularities. Thus, the L1 background, in terms of similarities in the morphological systems, improved the participants' abilities for gender acquisition, as they showed better recall and generalization. Therefore, a shift in attention to new, relevant features can improve L2 processing. This shift can be initially forced by explicit training, but it should be automatized for optimal performance. The results from Kempe and colleagues are very consistent with this model. Gender categorization was influenced by the prior knowledge of another language, with a similar morphological system inducing an attentional bias to the relevant aspects of the input. This bias could also underlie other reported L1 effects in L2 processing, and also explain why full-form storage is not the strategy always observed in L2 learners. The reverse tendency for decomposition, which 
could appear even greater than in L1 speakers of a particular language, can be observed in L2 learners with a highly inflected L1, such as Finnish or Hungarian (Portin \& Matti, 2001; Portin et al., 2008). On the other hand, attention can also be triggered by the intrinsic characteristics of the input. As we previously mentioned, some categories are in general more easily acquired than others, even in L1, and this difference can be due to the salience of the morphological marker (Ellis, 2008). Of course, L1 attentional shaping by previous learning and the automatic capture of attention by this latter saliency effect are not mutually exclusive options, but may both influence the learning process.

Finally, an important factor that clearly affects L2 learning and has received little attention is motivation. The lack of study on this factor is most likely due to the difficulty of studying it. Although none of the contributions in this issue explicitly study this effect, the differences in performance observed in some L2 groups may reflect this factor. It is important to consider that, although other reasons, aside from motivation, could explain differences between L2 groups, learning research in other fields has shown that emotional variables that play a role in motivation can interact with learning enhancing discrimination of previously indistinguishable stimuli both at the behavioral and cortical levels (Li, Howard, Parrish, \& Gottfried, 2008; Barkat, Poncelet, Landis, Rouby, \& Bensafi, 2008). This line of research may help to understand individual differences in SLA in the future.

Age of acquisition: maturational factors in brain development and consequences for learning

Finally, different contributions in this journal's issue address the influence of the age of acquisition (AoA) in morphosyntactic learning. L1 and L2 acquisition have common processing demands, and some output representations may be comparable in the two learning situations. However, there are important differences that may influence the strategies (i.e., explicit vs. implicit) used depending on the moment in development when L2 is acquired. 
Simultaneous bilinguals are certainly exposed to a different environment than heritage speakers or those who learn L2 after L1, early in infancy or later in adulthood. This point is, of course, essential for input-driven models, as it may determine the extent of the tuning to L1 characteristics, leading to eventual interference with L2 at the time of acquisition. However, AoA is also a determinant factor because brain maturation is not homogeneous during development. Grey-matter increase and myelination of the connections in various regions are achieved at different rates, with the prefrontal and parietal lobes developing particularly late (Diamond, 2002; Uylings, 2006; Huttenlocher, 2002). This variability constrains cognitive functions and thus influences the way acquisition proceeds (Casey, Giedd, \& Thomas, 2000; Diamond, 2002). As we have mentioned previously, executive functions and attention that rely on these brain structures seem to play an important role in adult L2 acquisition and in explicit learning. Therefore, these cognitive functions may not be fully developed in early SLA. This point is relevant if we think again about the greater sensitivity to AoA effects in learning grammar, including morphosyntactic versus lexical acquisition (Birdsong et al., 2001; Johnson et al., 1991; Weber-Fox et al., 1996). Two possibilities arise under this scenario and need to be further investigated. If executive functions and control of attention are not available in early SLA, but early SLA leads to more native-like acquisition of grammar, then these functions may not be necessary in early L2 acquisition and L1 as opposed to late L2 acquisition. The use of these functions would correspond to a compensatory strategy: instead of relying on implicit mechanisms, late L2 learners would need to involve executive control. The key point here resides in the timing of the maturation of prefrontal cortex because the other possibility might be that executive function is needed for grammatical acquisition in both first and second language acquisition. From this point of view, word acquisition would precede grammatical acquisition, which would rely on these later maturating functions controlled by the posterior prefrontal cortex. The prefrontal cortex 
maturation progresses from more posterior to more anterior regions that evolve until adolescence (Diamond, 2002). Thus, maturation of the more anterior prefrontal regions would allow the engagement of more explicit strategies than those engaged by attention and working memory maturating earlier and that do not need to be engaged explicitly.

The declarative/procedural model proposed by Ullman (2001b) is the only model introducing the idea of maturational constrains to explain the shift from procedural learning in L1 to declarative learning in L2 for grammatical acquisition. This claim is based on animal studies showing that procedural knowledge (Fredriksson, 2000; Walton et al. 1992; Wolansky et al., 1999) is subject to a critical period. However, the studies in this issue with his collaborators (Bowden et al.; Morgan-Short et al.) acknowledge that morphosyntactic rules acquired initially in L2 as declarative knowledge may become proceduralized with increased exposure and proficiency. This point is in contrast with similar proposals (Clahsen \& Felser, 2006; Clahsen et al., this issue), in which syntactic knowledge is claimed to remain lexicalized and depend on pragmatic and world knowledge, even at high proficiency levels.

Thus far, neuroimaging evidence on the perception and production of L2 shows increased activation in the same areas used for L1 and L2 processing and also a heightened engagement of areas needed for executive control in the PFC, including the dorsolateral (DLPFC, BA46/9) and ventrolateral PFC (VLPFC, BA45/47), the anterior cingulate (ACC), and the basal ganglia (see Abutalebi, 2008 for a review). It is quite remarkable that these areas overlap completely with those proposed for the explicit system (Ashby et al., 2005). The development of the PFC follows a posterior to anterior progression (Diamond, 2002), with the IFG developing before the VLPFC, which occurs before the DLPFC. Therefore, an alternative possibility to the procedural-system critical period constraint would be, as previously mentioned, that the maturational limitation favors the use of the implicit system until more anterior areas of the prefrontal cortex develop and could allow for more explicit, declarative 
learning. The use of intentional strategies may then suppress the use of the circuit engaged in an implicit system (Fletcher et al., 2005). Thus, brain and cognitive maturation may then be detrimental for those aspects, such as morphosyntactic acquisition, that are initially acquired by incidental learning.

Nevertheless, it is worth noting that this argument allows for the possibility that automatization and proceduralization can still occur at high proficiency and with intense practice in L2. However, these issues underscore the importance of distinguishing simultaneous, early, and late bilinguals, particularly at early stages of L2 acquisition, and of extending studies to look at the developmental progression and possible qualitative changes that may arise during the course of SLA. In this monograph, two of the studies interested in the acquisition of gender (Morgan-Short et al. and Kempe et al.) trained the subjects in an L2, respectively, BROCANTO2 (an artificial language) or Russian. Only Morgan-Short et al. report longitudinal information at low proficiency and at the end of training. As we previously commented, the behavioral and ERP measures at different moments of the learning process allowed them to observe qualitative differences suggesting a shift from declarative to procedural processing of morphosyntactic information only in the group of learners who received no explicit learning. Also, in this study, it would have been interesting to directly compare the ERP responses at low and high proficiency for correct sentences where no violation appears. Based on what has been detailed here, it might have been possible to observe if ERP components modulated by attention could appear in that comparison. After training, participants who learned gender agreement should show an enhancement in the components modulated by endogenous attention if the system is attentionally tuned by learning (Ellis, 2008). In a recent study, we observed that learning the rules embedded in an artificial language induced an enhanced positivity around $200 \mathrm{~ms}$ after word onset (P2) when ERPs were compared at the beginning and at the end of exposure to the language (De Diego- 
Balaguer, Toro, Rodriguez-Fornells, \& Bachoud-Levi, 2007). This modulation (P2) was interpreted as a shift in attention biasing language learning processes because several studies have shown enhancement of this component for the salient stimuli that cued the selection of information relevant for the task being performed (Luck \& Hillyard, 1994).

\section{Conclusions}

In general, the different models presented seem to suggest a less strict distinction between lexicalization and compositionality, with a significant interaction in the course of developing the L2 morphology. As in L1 morphosyntactic acquisition and processing, a neural network (including Broca's area (BA44), subcortical structures, and temporal regions) is necessary for L2 morphosyntactic acquisition. Nevertheless, in view of the psycholinguistic results, a theoretical view that encompasses a dynamic shift from posterior to more anterior areas in the course of learning seems to be a more plausible explanation, compared to a more dichotomic perspective. Factors such as the AoA and the strategies used in the learning process seem to help determine the dynamics of this progressive shift. More anterior prefrontal areas (i.e., the anterior cingulate cortex and the dorsolateral and ventrolateral prefrontal cortex) may interact with this classically described network to recruit executive functions, including working memory and control of attention. This view is derived from integrating the models with the evidence provided by the contributions coming from different languages. It is only when considering the contrasting results from different languages that we will be able to improve the models. This will make them applicable to languages with a variety of morphological systems and will help avoid confounding variables such as regularity and decomposition, which overlap in some languages.

Overall, the contributions from this issue have provided important points for further exploration. Other cognitive functions, such as executive functions and, particularly, attention 
allocation, seem to have an important influence on the acquisition of L2 and may partially explain individual differences observed during acquisition. However, specific questions related to morphosyntactic processing, such as the influence of the similarity between the L1 and L2 languages in the way cognitive control is exerted, need to be further developed. Because this similarity can arise at different levels of processing, as proposed in Clahsen's comment (in this issue), one way to advance our understanding of this question would be to adopt the distinction between the abstract lemma level and the form lexeme level that exists in current psycholinguistic models of perception and production.

On the other hand, although the results across the studies presented here do not all agree, most likely due to the multifactorial nature of the issue addressed, implicit and explicit training seem to influence the way that morphosyntactic information is represented. The temporal progression of the usage of one learning system throughout the course of learning is a key question that could yield important insights about the learning process itself. Different contributions also show the influence of L1 morphosyntactic characteristics in the acquisition of L2. Several possibilities have been proposed for the way that L1 transfer is performed, but no clear answer is yet available. As we have seen, the results from these areas are also critical to improving our understanding of the underlying brain networks and dynamics in L2 acquisition, as well as the symptoms observed in bilingual aphasia.

Acknowledgements. This work has been supported by the Spanish Government (Ministerio de Ciencia e Innovación, programs PSI2008-03885 and PSI2008-03901). We are thankful to Kira Gor for her helpful comments and suggestions on a previous version of the manuscript. 


\section{References}

Abutalebi, J. (2008). Neural aspects of second language representation and language control. Acta Psychologica, 128, 466-478.

Alexander,G. E., DeLong,M. R., \& Strick,P. L. (1986). Parallel organization of functionally segregated circuits linking basal ganglia and cortex. Annual Review of Neuroscience, 9, 357381.

Amunts, K., Schleicher, A., Burgel, U., Mohlberg, H., Uylings, H. B., \& Zilles, K. (1999).

Broca's region revisited: cytoarchitecture and intersubject variability. The Journal of Comparative Neurology, 412, 319-341.

Ashby, F. G., \& O'Brien, J. B. (2005). Category learning and multiple memory systems. Trends in Cognitive Sciences, 9, 83-89.

Ashby, F. G., \& Valentin, V. V. (2005). Multiple systems of perceptual category learning: theory and cognitive tests. In H. Cohen \& C. Lefevbre (Eds.), Handbook of Categorisation in cognitive science (pp. 548). Oxford, UK: Elsevier.

Baddeley, A., Gathercole, S., \& Papagno, C. (1998). The phonological loop as a language learning device. Psychological Review, 105, 158-173.

Barkat, S., Poncelet, J., Landis, B. N., Rouby, C., \& Bensafi, M. (2008). Improved smell pleasantness after odor-taste associative learning in humans. Neuroscience Letters, 434, 108112.

Beretta, A., Campbell, C., Carr, T. H., Huang, J., Schmitt, L. M., Christianson, K., \& Cao, Y. (2003). An ER-fMRI investigation of morphological inflection in German reveals that the 
brain makes a distinction between regular and irregular forms. Brain and Language, 85, 6792.

Bialystok, E., \& Martin, M. M. (2004). Attention and inhibition in bilingual children: evidence from the dimensional change card sort task. Developmental Science, 7, 325-339.

Birdsong, D. (2006). Age and second language acquisition and processing: A selective overview. Language Learning, 56, 9-49.

Birdsong, D., \& Molis, M. (2001). On the evidence for maturational constraints in secondlanguage acquisition. Journal of Memory and Language, 44, 235-249.

Cantone, K. F. (2007). Code-switching in bilingual children. Dordrecht: Springer.

Casey, B. J., Giedd, J. N., \& Thomas, K. M. (2000). Structural and functional brain development and its relation to cognitive development. Biological Psychology, 54, 241-257.

Clahsen, H. (1999). Lexical entries and rules of language: a multidisciplinary study of German inflection. Behavioral and Brain Sciences, 22, 991-1013.

Clahsen, H. \& Felser, C. (2006). Grammatical processing in language learners. Applied Psycholinguistics, 27, 3-42

Clark, E. V. (1998). Morphology in Language Acquisition. In A. Spencer \& A. M. Zwicky (Eds.), The Handbook of Morphology (pp. 374-389). Oxford: Blackwell Publishers.

Costa, A., Hernandez, M., \& Sebastian-Galles, N. (2008). Bilingualism aids conflict resolution: evidence from the ANT task. Cognition, 106, 59-86.

Dahlin, E., Neely, A. S., Larsson, A., Backman, L., \& Nyberg, L. (2008). Transfer of learning after updating training mediated by the striatum. Science, 320, 1510-1512.

Daneman, M., \& Carpenter, P. A. (1980). Individual differences in working memory and reading. Journal of Verbal Learning and Verbal Behavior, 19, 450-466. 
De Diego-Balaguer, R., Couette, M., Dolbeau, G., Durr, A., Youssov, K., \& Bachoud-Levi, A. C. (2008). Striatal degeneration impairs language learning: evidence from Huntington's disease. Brain, 131, 2870-2881.

De Diego-Balaguer, R., Rodriguez-Fornells, A., Rotte, M., Bahlmann, J., Heinze, H. J., \& Munte, T. F. (2006). Neural circuits subserving the retrieval of stems and grammatical features in regular and irregular verbs. Human Brain Mapping, 27, 874-888.

De Diego-Balaguer, R., Toro, J. M., Rodriguez-Fornells, A., \& Bachoud-Levi, A. C. (2007). Different neurophysiological mechanisms underlying word and rule extraction from speech. PLoS ONE, 2, e1175.

De Diego-Balaguer, R., \& Lopez-Barroso, D. (2009). Cognitive and neural mechanisms sustaining rule learning from speech. Language Learning (in press).

DeKeyser, R. M. (2000). The robustness of critical period effects in second language acquisition. Studies in Second Language Acquisition, 22, 499-533.

Diamond, A. (2002). Normal development of prefrontal cortex from birth to young adulthood: cognitive functions, anatomy, and biochemistry. In D. T. Stuss \& R. T. Knight (Eds.), Principles of Frontal Lobe Function (pp. 466-503). New York: Oxford University Press.

Dominey, P. F., Hoen, M., Blanc, J. M., \& Lelekov-Boissard, T. (2003). Neurological basis of language and sequential cognition: evidence from simulation, aphasia, and ERP studies. Brain and Language, 86, 207-225.

Dominey, P. F., Inui, T., \& Hoen, M. (2008). Neural network processing of natural language: II. Towards a unified model of corticostriatal function in learning sentence comprehension and non-linguistic sequencing. Brain and Language, 109, 80-92

Ellis, N. (2008). Usage-based and form-focused language acquisition: the associative learning of constructions, learned-attention and the limited L2 endstate. In P. Robinson \& N. C. Ellis 
(Eds.), Handbook of Cognitive Linguistics and Second Language Acquisition (pp. 372-405).

New York: Taylor \& Francis

Filoteo, J. V., Maddox, W. T., Salmon, D. P., \& Song, D. D. (2005). Information-integration category learning in patients with striatal dysfunction. Neuropsychology, 19, 212-222.

Fletcher, P. C., Zafiris, O., Frith, C. D., Honey, R. A., Corlett, P. R., Zilles, K., \& Fink, G.R. (2005). On the benefits of not trying: brain activity and connectivity reflecting the interactions of explicit and implicit sequence learning. Cerebral Cortex, 15, 1002-1015.

Hauser, M. D. \& Bever, T. (2008). A biolinguistic agenda. Science, 322, 1057-1059.

Hickok, G., \& Poeppel, D. (2004). Dorsal and ventral streams: a framework for understanding aspects of the functional anatomy of language. Cognition, 92, 67-99.

Hickok, G., \& Poeppel, D. (2007). The cortical organization of speech processing. Nature Reviews Neuroscience, 8, 393-402.

Hillis, A. E., Barker, P. B., Wityk, R. J., Aldrich, E. M., Restrepo, L., Breese, E. L., \& Work, M. (2004). Variability in subcortical aphasia is due to variable sites of cortical hypoperfusion. Brain and Language, 89, 524-530.

Hoen, M., Golembiowski, M., Guyot, E., Deprez, V., Caplan, D., \& Dominey, P. F. (2003). Training with cognitive sequences improves syntactic comprehension in agrammatic aphasics. Neuroreport, 14, 495-499.

Huttenlocher, P. R. (2002). Neural plasticity. Cambridge, MA.: Harvard University Press.

Hyltenstam, K., \& Abrahamsson, N. (2000). Who can become native-like in a second language? All, some, or none? On the maturational constraints controversy in second language acquisition. Studia Linguistica, 54, 150-166. 
Joanisse, M. F., \& Seidenberg, M. S. (1999). Impairments in verb morphology after brain injury: A connectionist model. Proceedings of the National Academy of Sciences of the United States of America, 96, 7592-7597.

Johnson, J. S. \& Newport, E. L. (1991). Critical period effects on universal properties of language: the status of subjacency in the acquisition of a second language. Cognition, 39, 215258.

Kaan, E., Harris, A., Gibson, E., \& Holcomb, P. (2000). The P600 as an index of syntactic integration difficulty. Language and Cognitive Processes, 15, 159-201.

Koechlin, E., Danek, A., Burnod, Y., \& Grafman, J. (2002). Medial prefrontal and subcortical mechanisms underlying the acquisition of motor and cognitive action sequences in humans. Neuron, 35, 371-381.

Kotz, S. A., Frisch, S., Werheid, K., Hein, G., von Cramon, D. Y., \& Friederici, A. D. (2002). The role of the basal ganglia in syntactic language processing: Event-related potential evidence from different patient populations and syntactic paradigms. Brain and Language, 83, $68-70$.

Kutas, M., \& Federmeier, K. D. (2000). Electrophysiology reveals semantic memory use in language comprehension. Trends in Cognitive Sciences, 4, 463-470

Li, W., Howard, J. D., Parrish, T. B., \& Gottfried, J. A. (2008). Aversive learning enhances perceptual and cortical discrimination of indiscriminable odor cues. Science, 319, 1842-1845.

Lieberman, P. (2000). Human language and our reptilian brain: the subcortical bases of speech, syntax and thought. Cambridge, MA: Harvard University Press. 
Liegeois, F., Baldeweg, T., Connelly, A., Gadian, D. G., Mishkin, M., \& Vargha-Khadem, F. (2003). Language fMRI abnormalities associated with FOXP2 gene mutation. Nature Neuroscience, 6, 1230-1237.

Marcus, G. F., Fernandes, K. J., \& Johnson, S. P. (2007). Infant rule learning facilitated by speech. Psychological Science, 18, 387-391.

Marslen-Wilson, W. D., \& Tyler, L. K. (2007). Morphology, language and the brain: the decompositional substrate for language comprehension. Philosophical Transactions of the Royal Society B: Biological Sciences, 362, 823-836.

Matzke, M., Mai, H., Nager, W., Rüsseler, J., \& Münte, T. (2002). The costs of freedom: an ERP study of non-canonical sentences. Clinical Neurophysiology, 113, 844-52.

McClelland, J. L., \& Patterson, K. (2003). Differentiation and integration in human language. Reply to Marslen-Wilson and Tyler. Trends in Cognitive Sciences, 7, 63-64.

Middleton, F.A., \& Strick, P.L. (2000). Basal ganglia output and cognition: evidence from anatomical, behavioral, and clinical studies. Brain and Cognition, 42, 183-200.

Milner, B., Squire, L. R., \& Kandel, E. R. (1998). Cognitive neuroscience and the study of memory. Neuron, 20, 445-468.

Miozzo, M. (2003). On the processing of regular and irregular forms of verbs and nouns: evidence from neuropsychology. Cognition, 87, 101-127.

Montrul, S., \& Slabakova, R. (2003). Competence similarities between native and near-native speakers. An investigation of the preterite-imperfect contrast in Spanish. Studies in Second Language Acquisition, 25, 351-398.

Munte, T. F., \& Kutas, M. (2008). Capitalizing on deep brain stimulation: thalamus as a language monitor. Neuron, 59, 677-679. 
Osterhout, L., McLaughlin, J., Pitkänen, I., Frenck-Mestres, C., \& Molinaro, N. (2006). Novice learners, longitudinal designs, and event-related potentials: a means for exploring the neurocognition of second language processing. Language Learning, 56, 199-230.

Pasupathy, A., \& Miller, E. K. (2005). Different time courses of learning-related activity in the prefrontal cortex and striatum. Nature, 433, 873-876.

Pinker, S. (1999). Words and rules: the ingredients of language. London: Weidenfeld \& Nicolson.

Plunkett, K., \& Marchman, V. (1991). U-shaped learning and frequency effects in a multilayered perception: implications for child language acquisition. Cognition, 38, 43-102.

Poeppel, D., \& Embick, D. (2005). Defining the relation between linguistics and neuroscience. In A. Cutler (Ed.), Twenty-first century psycholinguistics: Four cornerstones (pp. 103-118). Mahwah: Lawrence Erlbaum.

Portin, M., Lehtonen, M., Harrer, G., Wande, E., Niemi, J., \& Laine, M. (2008). L1 effects on the processing of inflected nouns in L2. Acta Psychologica, 128, 452-465.

Portin, M., \& Matti, L. (2001). Processing cost associated with inflectional morphology in bilingual speakers. Bilingualism: Language and Cognition, 4, 55-62.

Rodriguez-Fornells, A., Cunillera, T., Mestres-Misse, A., \& De Diego-Balaguer, R. (2009). Neurophysiological mechanisms involved in language learning in adults. Philosophical Transactions of the Royal Society B: Biological Sciences, (in press).

Rodriguez-Fornells, A., De Diego-Balaguer, R., \& Munte, T. F. (2006). Executive control in bilingual language processing. In M. Gullberg \& $\mathrm{P}$. Indefrey (Eds.), The cognitive neuroscience of second language acquisition (pp. 133-190). Oxford: Blackwell Publishing. 
Rodriguez-Fornells, A., Rotte, M., Heinze, H. J., Nosselt, T., \& Munte, T. F. (2002). Brain potential and functional MRI evidence for how to handle two languages with one brain. Nature, 415, 1026-1029.

Scheutz, M., \& Eberhard, K. (2004). Effects of morphosyntactic gender features in bilingual language processing. Cognitive Science, 28, 559-588.

Seidenberg, M. S., \& Joanisse, M. F. (2003). Show us the model. Trends in Cognitive Sciences, 7, 106-107.

Sonnenstuhl, I., Eisenbeiss, S., \& Clahsen, H. (1999). Morphological priming in the German mental lexicon. Cognition, 72, 203-236.

Squire, L. R., \& Knowlton, B. J. (2000). The medial temporal lobe, the hippocampus, and the memory systems of the brain. In M. S. Gazzaniga (Ed.), The new cognitive neurosciences (pp. 765-779). Cambridge, MA: MIT Press.

Stern, C. E., Corkin, S., Gonzalez, R. G., Guimaraes, A. R., Baker, J. R., Jennings, P. J., ... Rosen, B. R. (1996). The hippocampal formation participates in novel picture encoding: evidence from functional magnetic resonance imaging. Proceedings of the National Academy of Sciences, 93, 8660-8665.

Teicher, M. H., Anderson, C. M., Polcari, A., Glod, C. A., Maas, L. C., \& Renshaw, P. F. (2000). Functional deficits in basal ganglia of children with attention-deficit/hyperactivity disorder shown with functional magnetic resonance imaging relaxometry. Nature Medicine, 6, $470-473$

Teichmann, M., Dupoux, E., Kouider, S., Brugieres, P., Boisse, M. F., Baudic, S., ... Bachoud-Lévi, A-C. (2005). The role of the striatum in rule application: the model of Huntington's disease at early stage. Brain, 128, 1155-1167. 
Tomasello, M., \& Brooks, P. (1999). Early syntactic development: A construction grammar approach. In M. Barret (Ed.), The development of language (pp. 161-190). Hove: Psychology Press.

Ullman, M. T. (2001a). The neural basis of lexicon and grammar in first and second language: The declarative/procedural model. Bilingualism: Language and Cognition, 4, 105-122.

Ullman, M. T. (2001b). A neurocognitive perspective on language: The declarative/procedural model. Nature Reviews Neuroscience, 2, 717-726.

Ullman, M. T. (2006). Is Broca's area part of a basal ganglia thalamocortical circuit? Cortex, $42,480-485$.

Ullman, M. T., Corkin, S., Coppola, M., Hickok, G., Growdon, J. H., Koroshetz, W. J., \& Pinker, S.. (1997). A neural dissociation within language: Evidence that the mental dictionary is part of declarative memory, and that grammatical rules are processed by the procedural system. Journal of Cognitive Neuroscience, 9, 266-276.

Uylings, H. B. M. (2006). Development of the human cortex and the concept of "critical" or "sensitive" periods. In M.Gullberg \& P. Indefrey (Eds.), The cognitive neuroscience of second language acquisition (pp. 59-90). Oxford: Blackwell Publishing.

Wahl, M., Marzinzik, F., Friederici, A. D., Hahne, A., Kupsch, A., Schneider, G. H., ... Klostermann, F. (2008). The human thalamus processes syntactic and semantic language violations. Neuron, 59, 695-707.

Weber-Fox, C. M., \& Neville, H. J. (1996). Maturational constraints on functional specialization for language processing: ERP and behavioral evidence in bilingual speakers. Journal of Cognitive Neuroscience, 8, 231-256.

Yin, H. H., \& Knowlton, B. J. (2006). The role of the basal ganglia in habit formation. Nature Reviews Neuroscience, 7, 464-476. 


\section{FIGURE LEGENDS}

Figure 1. A. Representation of the Declarative/Procedural model (Ullman, 2001). Declarative memory is represented on the left. It comprises storage of semantic and episodic (explicit) knowledge. The circuit proposed for procedural (implicit) memory is represented on the right side. B. Representation of the COVIS explicit system for categorization (adapted from Ashby et al., 2005). Sharp-black arrow endings represent excitatory connections. Round-red endings represent inhibitory connections. The green arrow indicates a dopaminergic projection (ACC $=$ anterior cingulate cortex DA $=$ Dopaminergic projection; $\mathrm{PFC}=$ Prefrontal Cortex $). \mathrm{C}$. Results for the comparison between present tense regular (cant-ar $\rightarrow$ cant- $o$ ) and irregular (sent-ir $\rightarrow$ sient- $o$ ) covert production of Spanish verbs (adapted from de Diego-Balaguer et al., 2006) versus repetition conditions. Within PFC, a dissociation between the anterior and posterior prefrontal regions was observed for irregular-regular verbs. The irregular condition, requiring the retrieval of the appropriate stem showed more activation in anterior prefrontal regions (dorsolateral prefrontal cortex, BA46 and anterior inferior frontal gyrus, BA45). Regular verbs, requiring the maintenance of the same stem presented, showed more activation in the posterior inferior frontal gyrus (opercular region, BA44) at the border with the anterior superior temporal gyrus. 